\title{
Satisfacción residencial en un barrio remodelado: Predictores fisicos y sociales
}

\author{
MARÍA AMÉRIGO y JUAN IGNACIO ARAGONÉS \\ Universidad Complutense de Madrid
}

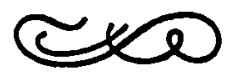

Resumen

El trabajo que aqui se presenta consiste en una investigación llevada a cabo en un ärea de remodelación, con dos objetivos primordiales: Por un lado, comprobar en que medida las categorias de acasan, barrios y evecinoss son dimensiones importantes para el estudio de satisfacción residencial; y en segundo lugar, encontrar predictores fisicos y sociales de dicho constructo a través de un instrumento válido a tal fin. Los resultados obtenidos en una muestra de 232 amas de casa, confirman la relevancia de las categorias mencionadas anteriormente. En cuanto al segundo objetivo, un análisis de regresión multiple pone de manifiesto que son cuatro los factores que más expli. can la satisfacción residencial en este área concreta, siendo el caislamiento térmicos el más importante de ellos.

\section{Abstract}

This paper is an investigation carried out in a redevelopment area with two chief aims. Firstly,

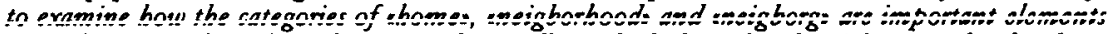
in studying resindential satisfaction; and secondly, to find physical and social aspects for the above mentioned residential satisfaction by using questionnaire. The results obtain from survey 232 bouswives, confirm the relevance of the above mentioned categories. As for second, a study of multiple regresion demostrates there are four factors. Which primarily influence residential satisfaction in this area the most important of which insulation from the temperature.

Dirección del autor: Departamento de Psicología Social. Campus de Somosaguas. Universidad Complutense de Madrid. 
En los últimos tiempos aparecen multitud de investigaciones en lengua inglesa en las que se trabaja sobre indicadores subjetivos de calidad de vida o de bienestar. Uno de los temas que surgen dentro de este grupo de trabajo, son los correspondientes a la satisfacción residencial con fuerte implantación dentro de la Psicología Ambiental. Recientes revisiones sobre el tema pueden encontrarse en Weidemann y Anderson (1985), y Aragonés y Amérigo (1987).

En la mayoría de los trabajos puede notarse la ausencia de un marco teórico que explique cómo opera este constructo. Tan sólo modelos sistémicos como los de Marans y Rodgers (1975), y Weidemann y Anderson (1985), merecen destacarse entre la bibliografía. El resto de trabajos responden a estudios diferenciales de satisfacción residencial, o a lo sumo, a la búsqueda de predictores de satisfacción residencial en ambientes muy específicos, siendo en gran cantidad de casos las casas subvencionadas el objetivo central de las investigaciones. La razón de que tales contextos sean objeto de estudio se debe, muy probablemente, a la intervención en la construcción de la Administración y al mucho capital invertido, aunque no debe olvidarse que de hecho afecta a muchas familias.

La satisfacción residencial tal y como se viene entendiendo dentro de la Psicología Ambiental, corresponde a un concepto de tipo actitudinal en el que debe tenerse en cuenta la influencia del rol ambiental. es decir, «los aspectos del rol organizacional o social de una persona que se relacionan con su trato con el ambiente físico», tal y como demuestran Canter y Walker (1980, p. 30).

La investigación que se presenta en estas páginas pretende ser un acercamiento a esta problemática desde dos vertientes diferentes: por un lado, comprobar en qué medida la satisfacción residencial es función de las categorías barrio, casa y vecinos, estudiadas por Canter y Rees (1984); y en segundo término los predictores físicos y sociales de un barrio periférico de Madrid de estatus socioeconómico bajo.

\section{METODOLOGIA}

\section{Definición del marco ambiental}

El estudio en cuestión se realizó en una de las tres zonas que componen la barriada de Orcasitas, concretamente en Poblado Dirigido. Las edificaciones en este área se llevaron a cabo en los años cincuenta, terminándose de construir en 1960. Catorce años más tarde, serios problemas estructurales comenzaron a aparecer, tales como grietas de considerable extensión, etc. que hicieron agruparse a los usuarios para denunciar el estado de sus viviendas que claramente se decantaba como ruinoso.

Tras varias luchas y reivindicaciones consiguieron que en el año 1979 se llevara a cabo la remodelación, en la que se ha respetado aproximadamente la ubicación anterior tanto de los bloques de pisos como de las viviendas unifamiliares, por lo que, en su conjunto, la estructura arquitectónica actual es similar a la que había en un principio. La remodelación finalizó en 1984.

$\mathrm{La}$ adjudicación de viviendas depende del Instituto de la Vivienda de Madrid (IVIMA), por lo que son de carácter público. En concreto, el Poblado Dirigido de Orcasitas está formado por un total de 2.953 viviendas, de las cuales 1.920 corresponden a pisos en altura y 1.033 a viviendas unifamiliares. 
Los bloques de pisos están compuestos por diez alturas, en cada una de las cuales se sitúan dos viviendas, que en unos casos son de tres dormitorios y en otros de cuatro. Dichos bloques están ubicados de tal forma que encierran jardines interiores de los que se ocupan los propios usuarios. La zona está dotada de un conjunto comercial, polideportivo, ambulatorio, iglesia y de un local para la Asociación de Vecinos donde también se realizan actividades culturales.

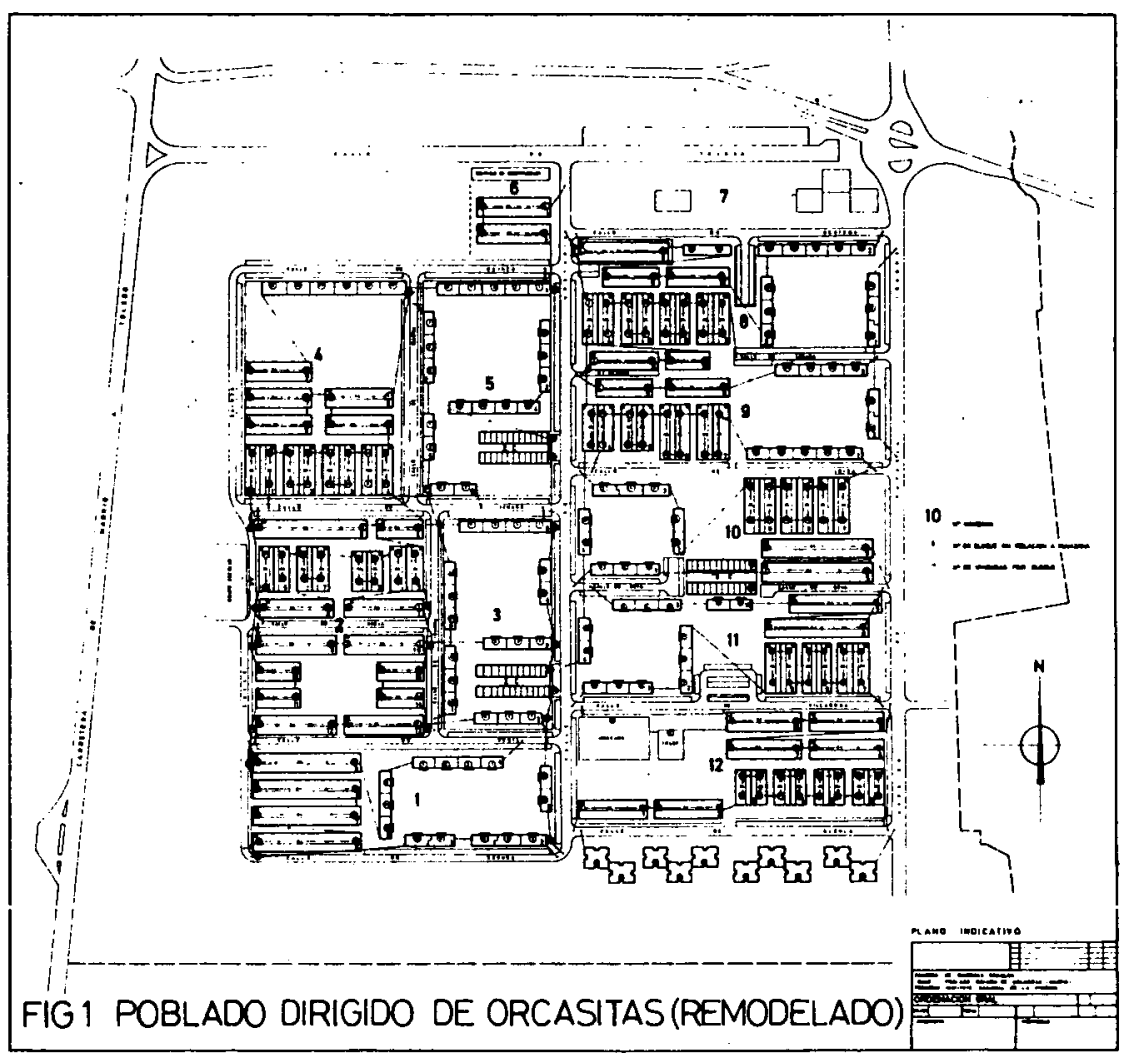

\section{Sujetos}

La muestra estudiada está compuesta por 232 amas de casa, residentes en bloques de pisos, elegidas al azar por un sistema de rutas. Aunque el abanico de edad está comprendido entre los 19 y 82 años, más del $65 \%$ de la muestra tiene una edad superior a los 50 años, porcentaje tan alto debido a que forman parte de la población original. En cuanto al nivel de estudios, el $62 \%$ posee sólo estudios primarios y el $25 \%$ no tiene estudios.

Esta muestra está obtenida de una población muy homogénea con características sociodemográficas similares -estatus socioeconómico y nivel cultural bajos-y con una experiencia común: existe un gran lazo de unión entre los vecinos, promovido por las luchas y reivindicaciones argüidas anteriormente. Por otra parte, la remodelación apenas ha influido en las redes sociales del barrio, debido a que los usuarios fueron asignados a condiciones de vivienda (proximidad, altura, orientación), muy similares a las anteriores, de tal forma que la familia que en un principio vivía en casa baja, se le asignaba una vivienda 
unifamiliar y exactamente lo mismo con familias que vivían en bloques de pisos, incluso respetando, en este último caso, aproximadamente, la altura original.

\section{Técnica}

Con el fin de averiguar cuáles son los predictores físicos y sociales de la satisfacción residencial en los estatus socioeconómicos bajos, se trató de confeccionar un instrumento que supusiera un primer intento para medir la satisfacción residencial en poblaciones de casas adjudicadas por la Administración, cuyo requerimiento ya fue argumentado por J. A. Corraliza (1987).

El instrumento utilizado para esta investigación es un cuestionario compuesto por 58 ítems, 43 de los cuales están destinados a obtener predictores de satisfacción y están formulados siguiendo una escala Likert de cinco puntos, desde muy de acuerdo a muy en desacuerdo. Para evitar la forma de contestar rutinaria, se intercalaron hacia la mitad de esta serie de 43 ítems, cuatro preguntas sin efectos de cara a los objetivos de esta investigación. Seguidamente fueron incluidos 4 ítems referidos a la satisfacción con el barrio, la vivienda, los vecinos y en general, formulados en una escala Likert de siete puntos, desde muy satisfecha hasta muy insatisfecha. El resto de los ítems están referidos a variables sociodemográficas recogidas en la literatura sobre este campo, tales como edad, número de hijos, edad del hijo más pequeño, existencia o no de familiares en el barrio, etc.

La elaboración de los 43 ítems iniciales se realizó partiendo de cuarenta y nueve entrevistas estructuradas, de las cuales veintisiete correspondían a sujetos de clase baja y veintidós a sujetos de clase media y media-alta.

El objetivo de esta entrevista era cubrir aspectos positivos y negativos sobre la calidad residencial y, en concreto, sobre tres aspectos claves que Canter y Rees (1984) señalan en su estudio sobre la satisfacción residencial: barrio, casa y vecinos.

Posteriormente, las entrevistas fueron sometidas a un análisis de contenido. Los ítems se elaboraron a partir de aquellas categorías que obtuvieron mayor frecuencia. Se intentó formular dos ítems por cada categoría, de tal forma que uno recogiera un aspecto cognitivo y el otro uno conductual de la misma; tarea en muchos casos limitada por el carácter específico de algunas categorías. Tanto la formulación positiva o negativa del ítem como su posición en la escala, se realizaron de forma aleatoria resultando 21 ítems negativos y 22 positivos.

Una vez elaborado el cuestionario se procedió a su cumplimentación a través de un sistema aleatorio de rutas.

Es oportuno resaltar que dado el nivel cultural de los sujetos, el encuestador iba provisto de una serie de tarjetas que incluían la gama de respuestas y que eran entregadas el sujeto entrevistado con intención de facilitar su labor.

\section{Resultados}

Con el propósito de cubrir el primer objetivo de esta investigación, es decir, de ver en qué medida influían las categorías de barrio, vivienda y vecinos sobre la satisfacción en general, tal y como estudiaron Canter y Rees (1984), se procedió a un análisis de regresión múltiple para ver cuánto de satisfacción general era explicada por estas variables. Los resultados de este primer análisis se detallan en la tabla 1. 
TABLA I

Análisis de regresiōn múltiple de las categorias barrio, casa y vecinos sobre satisfacción

\begin{tabular}{|l|c|c|c|}
\hline & $\mathbf{R}^{2}$ & coef. reg. std & P \\
\hline BARRIO & .5024 & .465 & .01 \\
\hline CASA & .6282 & .331 & .01 \\
\hline VECINOS & .6619 & .218 & .01 \\
\hline
\end{tabular}

Como puede observarse, el análisis de regresión múltiple paso a paso (stepwise), pone de manifiesto que es la satisfacción con el barrio la variable que más explica la satisfacción en general, seguida de la satisfacción con la casa y finalmente con los vecinos. Las tres variables juntas explican una alto porcentaje de la varianza total en satisfacción $(66,19 \%)$.

Un segundo objetivo de este trabajo consistió en encontrar predictores de satisfacción. Para ello, se realizó un análisis factorial - máxima verosimilitud, rotación ortogonal- sobre los 43 ítems iniciales del cuestionario, obteniendo cinco factores que explican el $68,92 \%$ de la varianza total:

FACTOR 1: intimidad

FACTOR 2: confort con la unidad vecinal (2)

FACTOR 3: confortabilidad con la vivienda

FACTOR 4: seguridad

FACTOR 5: aislamiento térmico

La saturación de los ítems en cada factor se muestra en la tabla 2

TABLA II

Saturación de los ítems en los factores

\begin{tabular}{|c|c|c|c|c|c|}
\hline & \multicolumn{5}{|c|}{ FACTORES } \\
\hline & I & II & III & IV & $\vec{V}$ \\
\hline $\begin{array}{l}\text { Los vecinos de mi casa hacen ex- } \\
\text { cesivo ruido } \ldots \ldots \ldots \ldots \ldots\end{array}$ & .685 & & & & \\
\hline $\begin{array}{l}\text { En mi barrio hay demasiada } \\
\text { gente } \ldots \ldots \ldots \ldots \ldots \ldots\end{array}$ & .617 & & & & \\
\hline $\begin{array}{l}\text { Mis vecinos se meten mucho en } \\
\text { mi vida privada } \ldots \ldots \ldots \ldots\end{array}$ & .589 & & & & \\
\hline $\begin{array}{l}\text { En general puedo decir que mis } \\
\text { vecinos son mala gente ...... }\end{array}$ & .548 & & & & \\
\hline $\begin{array}{l}\text { Mis vecinos me ayudan siempre } \\
\text { que lo necesito } \ldots \ldots \ldots \ldots\end{array}$ & -.497 & & & & \\
\hline $\begin{array}{l}\text { Los vecinos de mi barrio en ge- } \\
\text { neral son gente amable...... }\end{array}$ & & .667 & & & \\
\hline $\begin{array}{l}\text { Mis vecinos y yo mantenemos } \\
\text { buenas relaciones .......... }\end{array}$ & & .572 & & & \\
\hline $\begin{array}{l}\text { Durante el día mi casa está bien } \\
\text { iluminada } \ldots \ldots \ldots \ldots \ldots \ldots\end{array}$ & & .569 & & & \\
\hline
\end{tabular}


T.ABL. II (continuación)

\begin{tabular}{|c|c|c|c|c|c|}
\hline & \multicolumn{5}{|c|}{ F A C TORES } \\
\hline & $\mathrm{I}$ & II & III & IV & $\mathrm{V}$ \\
\hline $\begin{array}{l}\text { En mi barrio hay suficientes par- } \\
\text { ques } y \text { jardines } \ldots \ldots \ldots \ldots \ldots\end{array}$ & & .533 & & & \\
\hline $\begin{array}{l}\text { Mi casa está bien dotada de ar- } \\
\text { marios empotrados } \ldots . . . .\end{array}$ & & .531 & & & \\
\hline $\begin{array}{l}\text { El transporte de mi barrio me } \\
\text { permite desplazarme con } \\
\text { facilidad } \ldots \ldots \ldots \ldots \ldots \ldots \ldots\end{array}$ & & .460 & & & \\
\hline $\begin{array}{l}\text { La asociación de vecinos es efi- } \\
\text { caz de cara a resolver los proble- } \\
\text { mas del barrio } \ldots \ldots \ldots \ldots \ldots \ldots\end{array}$ & & .460 & & & \\
\hline $\begin{array}{l}\text { El barrio tiene suficientes insta- } \\
\text { laciones deportivas.......... }\end{array}$ & & .416 & & & \\
\hline 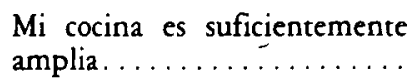 & & & .745 & & \\
\hline $\begin{array}{l}\text { Mi vivienda tiene un buen cuar- } \\
\text { to de baño } \ldots \ldots \ldots \ldots \ldots\end{array}$ & & & .645 & & \\
\hline $\begin{array}{l}\text { Puedo decir que mi casa tiene } \\
\text { una buena ventilación } \ldots . . .\end{array}$ & & & .585 & & \\
\hline Mi barrio está en ruinas & & & -.445 & & \\
\hline $\begin{array}{l}\text { Mi casa en general es cómoda y } \\
\text { confortable } \ldots \ldots \ldots \ldots \ldots\end{array}$ & & & .422 & & \\
\hline $\begin{array}{l}\text { En mi barrio existe mucha } \\
\text { delincuencia } \ldots \ldots \ldots \ldots \ldots\end{array}$ & & & & .783 & \\
\hline $\begin{array}{l}\text { Las madres pueden estar tran- } \\
\text { quilas dejando jugar a los niños } \\
\text { solos en la calle } . . . \ldots \ldots \ldots\end{array}$ & & & & -.583 & \\
\hline $\begin{array}{l}\text { La droga es un problema funda- } \\
\text { mental en mi barrio } \ldots \ldots \ldots \ldots \\
\text { La calefacción de mi casa calien- } \\
\text { ta poco } \ldots \ldots \ldots \ldots \ldots \ldots \ldots\end{array}$ & & & & .546 & .409 \\
\hline $\begin{array}{l}\text { Realmente los pisos tienen bue- } \\
\text { na calidad de construcción ... }\end{array}$ & & & & & -.433 \\
\hline Mi casa está aislada del frío.. & & & & .433 & \\
\hline Varianza que explica cada factor & 6.7276 & 1.9263 & 1.5730 & 1.4169 & 1.0718 \\
\hline
\end{tabular}

Posteriormente se procedió a estudiar cuál era la distribución de dichos factores en función de su mayor explicación sobre la varianza en satisfacción. Para ello se realizó un análisis de regresión múltiple paso a paso, cuyos resultados se muestran en la tabla 3 .

Como puede observarse, el factor 1 -intimidad - no aparece en el análisis, lo que permite pensar que su contribución a la satisfacción general no es significativa es este caso. Los cuatro factores restantes explican un $15,28 \%$ de la varianza total en satisfacción, destacando el factor 5 -aislamiento térmico- que explica el $13,37 \%$ de la varianza, y los factores 2 y 4, con una mínima contribución.

Añadidas al analisis de regresión la variables sociodemográficas: edad del sujeto encuestado, tener familiares en el barrio, número de hijos y edad del más 
TABLA III

Análisis de regresión mültiple de los factores sobre satisfacción

\begin{tabular}{|l|c|c|l|}
\hline & $\mathbf{R}^{2}$ & Incremento & \multicolumn{1}{c|}{$\mathbf{P}$} \\
\hline FACTOR 5 & .1337 & .1337 & .01 (Aislamiento térmico) \\
\hline FACTOR 2 & .1471 & .0134 & 01 (Confort con la unidad vecinal) \\
\hline FACTOR 3 & .1503 & .0032 & 01 (Confort con la vivienda) \\
\hline FACTOR 4 & .1528 & .0025 & 01 (Seguridad) \\
\hline
\end{tabular}

pequeño, se obtiene un coeficiente de correlación múltiple al cuadrado ligeramente superior al obtenido sólo con los cinco factores $\left(\mathrm{R}^{2}=.1736\right)$

\section{TABLA IV}

Análisis de regresión múltiple de los factores $y$ variables sociodemogräficas sobre la satisfacción

\begin{tabular}{|c|c|c|l|}
\hline & $\mathbf{R}^{2}$ & Coef. reg. std. & \multicolumn{1}{|c|}{$\mathbf{P}$} \\
\hline FACTOR 5 & .1126 & -.264 & .01 (Aislamiento térmico) \\
\hline EDAD & .1421 & .162 & .01 \\
\hline $\begin{array}{c}\text { PRESENCIA } \\
\text { FAMILIARES }\end{array}$ & .1581 & -.142 & .01 \\
\hline FACTOR 2 & .1736 & .125 & .01 (Confort con la unidad vecinal) \\
\hline
\end{tabular}

Entre los resultados merece destacarse de nuevo la importancia del factor 5 , así rome la de! factor 2 . TâñLiéñ iésaitan ias aportaciones a la satisfacción residencial de las variables edad y presencia de familiares en el barrio (ya destacado en los famosos trabajos del West End de Boston, Fried y Gleicher (1961), sobre todo cuando los sujetos eran de clase baja). No aparece, sin embargo, el número de hijos ni la edad del hijo más pequeño, contradiciendo la literatura sobre el tema que las consideran como variables con cierta significación de cara a explicar la satisfacción residencial (Hourihan, 1984).

A la vista de los resultados, los factores sólo explican un $15,28 \%$ de varianza, cantidad notoriamente menor que en las investigaciones sobre el tema, que vienen a ser del orden del $30 \%$ al. $50 \%$. Por otra parte el factor 5 -aislamiento térmico- es el que menos varianza explica en el cuestionario y sin embargo, el que más explica en satisfacción.

Esto plantea la necesidad de realizar un análisis discriminante que permita ver en qué medida los ítems del cuestionario son pertinentes al estudio, ya que la pretensión de esta investigación no sólo alcanza a señalar predictores de satisfacción, sino también un intento de establecer las bases de un cuestionario de satisfacción residencial que sea válido para estudiar este constructo en casas subvencionadas por la Administración para familias de estatus socioeconómico bajo.

Para realizar dicho análisis se definieron los grupos criterio utilizando como punto de corte la mediana en el ítem de satisfacción residencial general. Los resultados confirman que el cuestionario diseñado discrimina con 25 ítems (tabla 5) al $77,5 \%$ de los sujetos de la muestra (tabla 6) 
1. La calefacción de mi casa calienta poco.

2. Hay poca vigilancia en las calles de mi barrio.

3. Los servicios sanitarios del barrio cubren las necesidades básicas en cuanto a salud.

4. En mi barrio es fácil la convivencia entre payos y gitanos.

5. Mi barrio tiene gran cantidad de polución o contaminación.

6. Mi barrio está en ruinas.

7. Suelo ir a los parques y jardines de mi barrio.

8. Las calles están tan mal que no se puede ni andar.

9. Este barrio es muy ruidoso.

10. Mi barrio tiene problemas de aparcamiento.

11. En general puedo decir que mis vecinos son mala gente.

12. De vez en cuando en mi piso aparecen problemas de humedad.

13. Mi casa está mal aislada del frío.

14. En mi barrio hay suficientes colegios.

15. En mi barrio hay demasiada gente.

16. Mi casa en general es cómoda y confortable.

17. La asociación de vecios es eficaz de cara a resolver los problemas del barrio.

18. Realmente los pisos tienen buena calidad de construcción.

19. Mis vecinos me ayudan siempre que lo necesito.

20. La droga es un problema fundamental en mi barrio.

21. Mi barrio está bien surtido de tiendas.

22. El barrio tiene suficientes instalaciones deportivas.

23. Mi casa es realmente pequeña.

24. Los vecinos de mi barrio en general son gente amable.

25. Durante el día mi casa está bien iluminada.

TABLA VI

Análisis discriminante de los îtems sobre la satisfacción

\begin{tabular}{|c|c|c|c|c|}
\hline \multirow{2}{*}{} & \multicolumn{2}{|c|}{$\begin{array}{c}\text { PORCENTAJE } \\
\text { CORRECTO }\end{array}$} & \multicolumn{2}{c|}{ CLASIFICACION REAL } \\
\cline { 3 - 5 } & \multicolumn{2}{|c|}{ SAT } & + SAT \\
\hline \multirow{2}{*}{$\begin{array}{c}\text { Clasificación } \\
\text { Cuestionario }\end{array}$} & - SAT & 76.5 & 130 & 40 \\
\cline { 2 - 5 } & + SAT & 81.4 & 8 & 35 \\
\hline TOTAL & & 77.5 & 138 & 75 \\
\hline
\end{tabular}

- SAT: Puntuaciones que en el ítem de satisfacción tienen un valor menor o igual a 6 .

+ SAT: Puntuaciones que en el ítem de satisfacción tienen un valor mayor que 6. 


\section{DISCUSION}

En función de los resultados obtenidos, el primer objetivo relativo a la influencia sobre la satisfacción residencial de los tres aspectos claves recogidos en la literatura: barrio, casa, vecinos (Canter y Rees, 1984; Aragonés y Amérigo, 1987) ha quedado satisfactoriamente ratificado.

Los resultados apuntan que aún pudiendo ser el cuestionario eficaz para me. dir la satisfacción, la homogeneidad de la muestra y la implicación del vecinda. rio en la remodelación de su barrio, aconsejan buscar otras vías que contemplen esta problemática.

Por lo que respecta a los factores «confort en la vivienda» y «seguridad», merece destacarse la escasa aportación en la varianza total de satisfacción, si se tiene en cuenta que en las investigaciones tales como la de Weidemann et al (1982) los factores «evaluación del piso» y «seguridad», explicaban el $10 \%$ y el $3,2 \%$, respectivamente sobre la satisfacción residencial. Asimismo, Fried (1982), obtuvo en «seguridad», tras un análisis de regresión múltiple, el 5,7\% de la varianza en satisfacción.

En conclusión, los cinco factores están en consonancia con la investigación empírica sobre esta temática, pero la sistemática creación de cuestionarios ad boc hacen que sus etiquetas sean diferentes aunque apunten en la misma dirección.

En concreto, en esta investigación, el predictor más potente ha sido el aislamiento térmico, no emergiendo otros ya clásicos en la literatura y que contempla el cuestionario. La razón de tal resultado habría que buscarla en la homogeneidad de la muestra.en su nivel de satisfacción general y rol ambiental; y como señalan Weidemann y Anderson (1985), esto favorece la fuerte evaluación residencial positiva, y quiza este efecto atenúe la explicación de la satisfacción en función de los factores.

\section{Notas}

1 Agradecemos al equipo de seguimiento de remodelación de barrios del Instituto de la Vivienda de Madrid (IVIMA) el apoyo prestado en el trabajo de campo y a la profesora Rosario Martínez Arias por sus constantes comentarios a lo largo de esta investigación.

2 Este concepto hace referencia al utilizado por Lee (1968).

\section{Referencias}

ARAGONES. J.I. y AMERIGO, M. (1887). Satisfacción Residencial: un concepto de calidad de vida. Documentación Social, 133-154.

CANTER, D. y ReEs, K. (1984). Un Modelo Multivariado de Satisfacción con la Vivienda. Cuadernos de Psicología, 6, 146-171.

CANTER, D. y WALKER, E. (1980). Enviromental Role and Conceptualizations of Housing Journal of Architectural Research, 7, 30-35. 
CónRauza. J.A. (1987). La Experiencia del Ambiente. Percepción y Significado del Medio Construido. Madrid, Tecnos.

FRIED, M. (1982). Residential Attachment: Sources of Residential and Community Satisfaction. Journal of Social Issues, 38 107-119.

Fried, M. y GleICHER, P. (1961) Some Sources of Residential Satisfaction in Urban Slum. Joumal of American Institute of Planners, 27 305-315.

Houruhan, K., (1984). Contex-Dependent Models of Residential Satisfaction. An Analysis of Housing Groups in Cork, Ireland. Environment and Behavior, 16 369-393.

LEE, T.R. (1968). Urban Neighborhood as a Socio-Spatial Schema. Human Relations, 21, 241-267.

MARANS, R.W. y RODGERS, S.W., (1975). Toward and Understanding of Community Satisfaction. En: A. Hawley y V. Rock (Eds.). Metropolitan America in Contemporary Perspective. Nueva York, Halstead Press.

Weidemann, S. y Anderson, J.R. (1985). A Conceptual Framework for Residential Satisfaction. En: I. Altman y C. H. Werner (Eds.). Home Environments. Nueva York, Plenum Press, 153-182.

WeidemanN, S.; ANDerson. J.R.; ButTerfield, D.J. y O'donnell (1982). Residents Petceptions of Satisfaction and Safety. Basis for Change in Multifamily Housing. Environment and Behavior, $14,695-724$. 